\title{
A novel peptide targets CD105 for tumour imaging in vivo
}

\author{
XIAOLONG LI $^{1 *}$, XIAOFENG HUANG $^{1 *}$, JING ZHANG $^{1}$, HONGXING HUANG $^{2}$, \\ LONG ZHAO $^{3}$, MEI YU ${ }^{1}$, YAN ZHANG ${ }^{2}$ and HUA WANG ${ }^{1,3 *}$ \\ ${ }^{1}$ Department of Oral and Maxillofacial Surgery, Clinical Laboratory, Guanghua School of Stomatology, \\ Hospital of Stomatology, Sun Yat-sen University, Guangdong Provincial Key Laboratory of Stomatology, \\ Guangzhou, Guangdong 510055; ${ }^{2}$ Laboratory of Cancer and Stem Cell Biology, \\ Key Laboratory of Gene Engineering of The Ministry of Education, State Key Laboratory of Biocontrol, \\ School of Life Sciences, Sun Yat-sen University, Guangzhou Higher Education Mega Center, \\ Guangzhou, Guangdong 510006; ${ }^{3}$ Guangzhou Yidai Pharmaceutical Co., Ltd., \\ Guangzhou, Guangdong 510055, P.R. China
}

Received January 30, 2018; Accepted July 12, 2018

DOI: $10.3892 /$ or.2018.6643

\begin{abstract}
Non-antibody-binding proteins (nABPs) have attracted attention in the field of alternative antibody approaches due to their ability to penetrate cell membranes. CD105 has been demonstrated to be a useful marker for identifying angiogenic endothelial, mesenchymal stem and osteosarcoma cells. In the present study, a novel non-antibody-binding protein (namely nABP296) with a high affinity for recombinant human CD105 was identified by M13 phage biopanning. Fluorescence microscopy, flow cytometry and in vivo animal imaging were used to confirm the visualization effect of the novel peptide to the CD105-positive MNNG/HOS cell line in vivo and in vitro. Immunofluorescence of tissue sections was also used to identify the target efficiency of the peptide in tumour sections derived from an MNNG/HOS xenograft tumour model and osteosarcoma patients. This peptide specifically was found to bind to the CD105-positive osteosarcoma MNNG/HOS cell line and the osteosarcoma cells in the histological sections derived from an MNNG/HOS xenograft model and osteosarcoma patients in vitro. This peptide also successfully labelled an animal osteosarcoma xenograft model in vivo. Taken together, a novel peptide that can be used as a potent agent for CD105 molecular targeting was identified,
\end{abstract}

Correspondence to: Dr Hua Wang, Department of Oral and Maxillofacial Surgery, Clinical Laboratory, Guanghua School of Stomatology, Hospital of Stomatology, Sun Yat-sen University, 56 Lingyuan West Road, Yuexiu, Guangzhou, Guangdong 510055, P.R. China

E-mail: wanghua9@mail.sysu.edu.cn

*Contributed equally

Abbreviations: nABP, non-antibody-binding peptide; CCK-8, Cell Counting Kit-8; CLSM, confocal laser scanning microscopy

Key words: peptide, CD105, osteosarcoma, diagnosis, biomarker and this peptide can be applied for osteosarcoma visualization in vitro and in vivo.

\section{Introduction}

CD105, also referred to as endoglin, is a type I membrane glycoprotein that forms part of the TGF- $\beta$ receptor complex on cell membranes (1). CD105 is a $180 \mathrm{kDa}$ homodimeric transmembrane protein composed of 633 amino acids, which consists of a large extracellular domain, a hydrophobic transmembrane domain and a short intracellular domain (2). The CD105 external domain binds to the TGF- $\beta 1$ and TGF- $\beta 3$ isoforms with a high affinity, forming the TGF- $\beta$ receptor complex (3). It serves an important role in regulating cellular proliferation, differentiation, migration, adhesion and angiogenesis, which are vital processes for tumour growth, survival and metastasis $(4,5)$. CD105 is predominantly expressed in angiogenic endothelial cells, or blood vessels surrounding tumour tissues and mesenchymal stem cells (MSCs) (6-8). The CD105 antibody has been used to label tumour microvessel density, separate MSCs and conjugate drug for targeting therapy (9-11).

Osteosarcoma is one of the most common primary malignant bone sarcomas, predominantly targeting the long cylindrical bones. Approximately 5\% of all osteosarcomas are reported to occur in the maxillofacial region (12). This tumour is more prevalent in adolescents and children, with an incidence rate of 3-4.5 million individuals per year globally, and can aggressively metastasise to the lung in the early stages $(13,14)$. In a previous study, a subset of bone sarcoma cells that expressed the MSC marker CD105 was reported to be more aggressive in comparison with other bone sarcoma cell lineages (15). Therefore, the CD105 antibody may be a marker for identifying the malignancy of osteosarcoma.

The complexity of production, heat instability, and poor penetration to the cancer microenvironment has limited the application of antibodies in vivo. Non-antibody-binding protein (nABP) is a heat-stable, artificially synthesised peptide with a potent penetration effect to the tumour microenvironment due 
to its small size and positive charge (16). Owning to the advantages of this peptide, it has attracted previous attention and has been used in various antitumour studies and tumour-targeting therapies $(17,18)$.

In the present study, 13 novel peptides binding to CD105 were identified using M13 phage display. Among these 13 peptides, the nABP296 peptide had a higher affinity for the CD105-positive osteosarcoma MNNG/HOS cell line as compared with that of other peptides, and a lower affinity for the CD105-negative Cal27 cell line. In addition, nABP296 was used to visualise the tumour in a MNNG/HOS tumour-bearing mouse model. It was observed that nABP296 was able to label osteosarcoma histological sections derived from an animal MNNG/HOS xenograft tumour model and an osteosarcoma patient.

\section{Materials and methods}

Phage display biopanning. Phage display biopanning, an affinity selection technique that selects peptides binding to a given target, was conducted according to standard procedures (19). A peptide phage display library was commercially constructed (Ph.D.-12 Phage Display Peptide Library; New England Biolabs, Inc., Beverly, MA, USA) and used in subsequent experiments. The recombinant human CD105 protein (R\&D Systems, Inc., Minneapolis, MN) at $100 \mu \mathrm{g} / \mathrm{ml}$ in sterile phosphate-buffered saline (PBS) was added to individual sterilised MaxiSorp plates (Thermo Fisher Scientific, Inc., Waltham, MA, USA) and incubated overnight at $4^{\circ} \mathrm{C}$. The plate was washed six times with Tris-buffered saline $/ 0.1 \%$ Tween-20 (TBST) and then blocked for $1 \mathrm{~h}$ at $4^{\circ} \mathrm{C}$ using $1 \%$ bovine serum albumin (BSA) in PBS. M13 phage display libraries were allowed to bind for $1 \mathrm{~h}$ at room temperature, and the unbound phages were washed away 10 times with TBST. Subsequently, the binding phages were eluted by an elution buffer, and the eluted phages were then amplified for another round of biopanning. For each round of biopanning, the titering of M13 phage was calculated. The recovery rate was calculated as follows: Recovery rate $=$ Titering output $/$ titering input.

Phage ELISA binding assay. Subsequent to three rounds of biopanning, the target M13 phages were enriched. A total of 16 monoclonal phages were selected randomly for the ELISA binding essay. Recombinant human CD105 protein at $100 \mu \mathrm{g} / \mathrm{ml}$ in sterile PBS was added to 16 individual sterilised wells of MaxiSorp plates and incubated overnight at $4^{\circ} \mathrm{C}$. Additionally, 16 individual sterilised wells were coated using $1 \%$ BSA as control. The plate was washed six times with TBST and blocked for $1 \mathrm{~h}$ at $4^{\circ} \mathrm{C}$ using $1 \%$ BSA in PBS. Next, the 16 monoclonal phages were amplified and added to the individual wells with CD105 protein and 1\% BSA and incubated for $1 \mathrm{~h}$ at room temperature. Following repeated washing of the MaxiSorp ELISA plate with $0.1 \%$ TBST, bound phages were detected by incubation with mouse anti-M13 phage antibody (HRP conjugated; cat. no. ab6188; 1:20; Abcam, Cambridge, $\mathrm{UK}$ ). The ABTS $/ \mathrm{H}_{2} \mathrm{O}_{2}$ substrate (internally prepared) was used to measure the amount of bound HRP, and the absorbance was monitored at $405 \mathrm{~nm}$. The inserted DNA sequences of 13 positive affinity monoclonal phages were determined using the primer 5'-CCCTCATAGTTAGCGTAACG-3' (New England Biolabs, Inc.).

Synthesis of peptides. Based on aforementioned procedures, 13 peptides were synthesised through solid-phase peptide synthesis using Fmoc chemistry by Chinese Peptide Company (Jiangsu, China). An extra fluorescein-5-isothiocyanate (FITC) was linked at the amino $(\mathrm{N})$ terminus of all peptides for labelling. Prepared peptides were identified by mass spectrometry (MS), and the purity (>95\%) was assayed by high-performance liquid chromatography (HPLC; Fig. 1). The FITC-labelled peptides were stored at $-20^{\circ} \mathrm{C}$. For further usage, peptides were dissolved in sterilised water at $1 \mathrm{mg} / \mathrm{kg}$ and diluted according to the experimental requirements.

Determination of cell lines for binding assay. MNNG/HOS and Cal27 cell lines were obtained from the Type Culture Collection of the Chinese Academy of Sciences (Shanghai, China). MNNG/HOS cells were cultured in Dulbecco's modified Eagle's medium (DMEM)/F12 (Sigma-Aldrich; Merck KGaA, Darmstadt, Germany) supplemented with $5 \%$ foetal bovine serum (FBS), while Cal27 cells were cultured in DMEM (Sigma-Aldrich; Merck KGaA) supplemented with $10 \%$ FBS. The cells were plated onto $10 \mathrm{~cm}^{2}$ flasks and maintained at $37^{\circ} \mathrm{C}$ in $5 \% \mathrm{CO}_{2}$ with $90 \%$ relative humidity. In order to investigate the CD105 expression in MNNG/HOS and Cal27 cells, the cell lines were then examined by reverse transcription-polymerase chain reaction (RT-PCR), immunofluorescence and flow cytometry.

RT-PCR for CD105 expression measurement. A NanoDrop 1000 (Thermo Fisher Scientific, Inc., Wilmington, DE, USA) was used to measure the amount of RNA by spectrophotometry. A total of $300 \mathrm{ng}$ total RNA was extracted from tumour cells, and converted to complementary DNA using the ReverTra Ace ${ }^{\circledR}$ qPCR RT kit (Toyobo Life Science, Osaka, Japan) for PCR analysis. The semi-quantitative RT-PCR analysis was performed using the Takara Ex Taq ${ }^{\circledR}$ kit (Takara Bio, Inc., Otsu, Japan). The PCR reaction conditions were performed as follows: $95^{\circ} \mathrm{C}$ for $5 \mathrm{~min}$, and 30 cycles of $95^{\circ} \mathrm{C}$ for $30 \mathrm{sec}, 60^{\circ} \mathrm{C}$ for $30 \mathrm{sec}$ and $72^{\circ} \mathrm{C}$ for $1 \mathrm{~min}$, following by a final elongation at $72^{\circ} \mathrm{C}$ for $10 \mathrm{~min}$ and held at $16^{\circ} \mathrm{C}$ for $10 \mathrm{~min}$. The CD105 primers used were synthesised by Synbio Technologies (Suzhou, China) and were as follows: CD105 forward, 5'-CACCACAGCGGAAAAAGGTG-3' and reverse, 5'-GCCGGTTTTGGGTATGGGTA-3'; and GAPDH forward, 5'-AATGGGCAGCCGTTAGGAAA-3' and reverse, 5'-GCGCCCAATACGACCAAATC-3'). PCR products were then analysed by electrophoresis on a $1 \%$ agarose gel and visualised by ethidium bromide staining under UV illumination in Iquant Capture RT ECL (version 1.0.1; GE Healthcare, Chicago, IL, USA).

Immunofluorescence assay. Following collection in a $0.25 \%$ $(\mathrm{w} / \mathrm{v})$ trypsin solution with $0.025 \%(\mathrm{w} / \mathrm{v})$ ethylene diamine tetraacetic acid (EDTA), MNNG/HOS and Cal27 cells were seeded in a 6 -well flask with slides at a density of $1 \times 10^{5}$ cells/well and incubated at $37^{\circ} \mathrm{C}$ in $5 \% \mathrm{CO}_{2}$ with $90 \%$ relative humidity overnight. On the second day, the cells were fixed in $4 \%$ paraformaldehyde at room temperature for 
$15 \mathrm{~min}$, and non-specific binding sites were blocked by $5 \%$ BSA (Guangzhou Xiang Bo Biological Technology Co., Ltd., Guangzhou, China) for $1 \mathrm{~h}$ at room temperature. The cells were then incubated with the CD105 antibody (cat. no. MA1-19408; 1:200; Invitrogen; Thermo Fisher Scientific, Inc.) overnight at $4^{\circ} \mathrm{C}$. Unbound antibodies were eluted by washing three times with PBS. Subsequently, the cells were further treated with donkey anti-mouse IgG $(\mathrm{H}+\mathrm{L})$ highly cross-adsorbed Alexa Fluor 568 secondary antibody (cat. no. A10037; 1:1,000; Invitrogen; Thermo Fisher Scientific, Inc.) in blocking buffer for $1 \mathrm{~h}$ at room temperature. Following further washing with PBS, the nucleus was counterstained by DAPI, and the samples were visualised under confocal laser scanning microscopy (CLSM; Leica Microsystems GmbH, Wetzlar, Germany). For polypeptide labelling, the MNNG/HOS/HOS and Cal27 cells were incubated in polypeptide at a concentration of $100 \mu \mathrm{g} / \mathrm{ml}$ in $1 \mathrm{ml} \mathrm{PBS}$ for $30 \mathrm{~min}$ at $4^{\circ} \mathrm{C}$ prior to fixation.

Flow cytometry assay. Subsequent to collection in a $0.25 \%(\mathrm{w} / \mathrm{v})$ trypsin solution with $0.025 \%(\mathrm{w} / \mathrm{v})$ EDTA, MNNG/HOS and Cal27 cells were incubated with the CD105 antibody (cat. no. 130-102-819; 1:10; Miltenyi Biotec GmbH, Bergisch Gladbach, Germany) at $4^{\circ} \mathrm{C}$ for $10 \mathrm{~min}$. The cell pellets were washed three times in PBS, suspended in $500 \mu \mathrm{l}$ PBS and then analysed using a FACSCalibur flow cytometer (BD Biosciences, Franklin Lakes, NJ, USA). For polypeptides labelling experiments, the MNNG/HOS/HOS or Cal27 cells were incubated in $100 \mu \mathrm{g} / \mathrm{ml}$ peptides in $100 \mu \mathrm{lPBS}$ at $4^{\circ} \mathrm{C}$ for $30 \mathrm{~min}$. Subsequent to further washing with PBS, cell pellets were suspended in $500 \mu \mathrm{l}$ PBS and then analysed using a FACSCalibur flow cytometer.

ELISA binding assay. ELISA binding assay of nABP296 to CD105 protein was performed, with CD106 protein (R\&D Systems, Inc.) used as a control. Recombinant human CD105 protein and CD106 protein at a concentration of $100 \mu \mathrm{g} / \mathrm{ml}$ in sterile PBS were added to six individual sterilised MaxiSorp plates and incubated overnight at $4^{\circ} \mathrm{C}$. The plates were washed six times with TBST and blocked for $1 \mathrm{~h}$ at $4^{\circ} \mathrm{C}$ using $1 \%$ BSA in PBS. Subsequently, nABP296 peptide was added at a concentration of $100 \mu \mathrm{g} / \mathrm{ml}$ and incubated for $1 \mathrm{~h}$ at room temperature. The unbound peptide was removed by PBS washing for 10 times. The absorbance was measured using a VICTOR X5 Multilabel plate reader (PerkinElmer, Inc., Singapore) at $490 \mathrm{~nm}$, and the results are reported as the optical density.

Cytotoxicity assay. For the cytotoxicity assay, $1 \times 10^{5}$ cells/well were seeded in 96-well plates in $100 \mu 1$ complete culture medium. Subsequent to culturing overnight at $37^{\circ} \mathrm{C}$ in a $5 \%$ $\mathrm{CO}_{2}$ atmosphere, the cells were incubated in different concentrations of $\mathrm{nABP} 296$ solution ranging between 0.5 and $5 \mu \mathrm{Mol}$. At 24 and 48 h, $10 \mu \mathrm{l}$ Cell Counting Kit-8 (CCK-8; Dojindo Molecular Technologies, Inc., Kumamoto, Japan) solution was added to the cells and incubated for another $1 \mathrm{~h}$. The absorbance was then determined at $450 \mathrm{~nm}$ using the VICTOR X5 Multilabel plate reader, and the survival rate was calculated.

In vivo assay. Based on the aforementioned experiments, the nABP296 peptide was used in the in vivo experiment.
The animal use protocol was reviewed and approved by the Animal Ethical and Welfare Committee of Sun Yat-sen University (Guangzhou, Guangdong, China). Briefly, MNNG/HOS cells were subcutaneously transplanted into 5-week-old BALB/c Nod mice at $1 \times 10^{7}$ cells $/ \mathrm{ml}$ in DF. When the volume of the xenograft tumours reached $\sim 200 \mathrm{~mm}^{3}$, $1 \mathrm{mg} / \mathrm{ml} \mathrm{nABP} 296$ in $100 \mu \mathrm{l}$ sterile Milli-Q water was intravenously administered to the mice. The mice were observed using the IVIS Spectrum In Vivo Imaging system (PerkinElmer, Akron, OH, USA), and then images were captured after $1 \mathrm{~h}$ of administration. Next, the mice were euthanised, tumours were excised from the MNNG/HOS tumour-bearing mice and images were captured using the IVIS Spectrum In Vivo Imaging system. Tumours and hearts were freeze-sectioned into $6 \mu \mathrm{m}$ and fixed in $100 \%$ acetone for $15 \mathrm{~min}$. Following further washing with PBS, the nuclei of the sections were stained with DAPI. The results were analysed by CLSM (Leica Microsystems GmbH).

Histological staining. All patients participating in this experiment provided informed consent prior to tissue collection and agreed to the use of their samples in scientific research. The described experiments were approved by the Ethics Committee of The Hospital of Stomatology at Sun Yat-sen University (approval no. ERC-2017-11). A total of 2 patients (osteosarcoma patient: Male, 33 years old, hospitalised for 15 days in July 2017; tongue carcinoma patient: Male, 25 years old, hospitalised for 16 days in October 2017) who were treated in Guanghua School of Stomatology (Hospital of Stomatoloty, Sun Yat-sen University) and diagnosed with osteosarcoma and tongue carcinoma by the Department of Pathology were included in these experiments. Osteosarcoma and tongue carcinoma tissues were obtained from the patients during surgery.

Tissue slices derived from a xenograft tumour model, an osteosarcoma patient and a tongue carcinoma patient were used for histological staining. The tumour samples were sectioned $(6-\mu \mathrm{m}$ thick) using a cryostat microtome (Microm HM560; Thermo Fisher Scientific, Inc.). Non-specific binding sites were blocked by incubation with $5 \%$ BSA for $1 \mathrm{~h}$ at room temperature. Next, histological sections were incubated using a combination of $50 \mu \mathrm{Mol}$ nABP296 peptide and mouse monoclonal CD105 antibody (cat. no. MA1-19408; 1:200; Invitrogen; Thermo Fisher Scientific, Inc.) in blocking buffer at $4^{\circ} \mathrm{C}$ overnight. Unbound peptide and antibody were eluted by washing three times with PBS, following which cells were further treated with donkey anti-mouse IgG $(\mathrm{H}+\mathrm{L})$ highly cross-adsorbed Alexa Fluor 568 secondary antibody (cat. no. A10037; 1:1,000; Invitrogen; Thermo Fisher Scientific, Inc.) for $1 \mathrm{~h}$ at room temperature. Following further washing with PBS, the nucleus was stained with DAPI, and the results were analysed under CLSM (Leica Microsystems $\mathrm{GmbH}$ ).

Statistical analysis. Statistical analysis was performed using the GraphPad Prism software (version 5; GraphPad Software, Inc., La Jolla, CA, USA). One-way analysis of variance was used to evaluate the survival rate of MNNG/HOS cells at 24 and $48 \mathrm{~h}$. P-values of $<0.05$ were considered to denote statistically significant differences. 
Table I. Recovery rate of each round of biopanning.

\begin{tabular}{lrrc}
\hline Round & Input, pfu & Output, pfu & Ratio (output/input) \\
\hline 1st & $1.8 \times 10^{11}$ & $8.9 \times 10^{5}$ & $4.9 \times 10^{-6}$ \\
2nd & $1.56 \times 10^{11}$ & $2.4 \times 10^{6}$ & $1.54 \times 10^{-5}$ \\
3rd & $1.0 \times 10^{11}$ & $4.5 \times 10^{6}$ & $4.5 \times 10^{-5}$ \\
\hline
\end{tabular}

Recovery rates of three rounds of biopanning were calculated as titering ouput/titering input. pfu, plaque-forming unit.

\begin{abstract}
Results
Determining peptide binding to CD105. The recovery rate of three rounds of biopanning is shown in Table I. Subsequent to three rounds of biopanning, the recovery rate of M13 phage was raised from $4.9 \times 10^{-6}$ to $4.5 \times 10^{-5}$. M13 phage binding to recombinant human CD105 protein was enriched. To identify a monoclone phage that can bind to CD105, ELISA was performed to M13 phage single clones (A-P). A total of 13 monoclonal phages were confirmed to have high affinity for CD105, as shown in Table II. The sequences and characteristics of the 13 positive monoclonal phages are listed in Table III in the range between nABP295 and nABP307.
\end{abstract}

Peptide labelling of the CD105-positive MNNG/HOS cell line. Based on the results of RT-PCR, fluorescence and flow cytometry (Fig. 1), the MNNG/HOS cell line was found to be CD105-positive and the Cal27 cell line was observed to be CD105-negative. These two cell lines were then used in subsequent experiments to identify the peptide with high binding affinity for CD105 expressed in the cell membranes.

By flow cytometry, two peptides, namely nABP296 and nABP297, with high affinity for the CD105-positive MNNG/HOS cell line were identified (Fig. 2A and B). To determine whether these two peptides had a specific affinity for CD105, the binding of nABP296 and nABP297 to the CD105-positive MNNG/HOS and CD105-negative Cal27 cell lines was analysed by a flow cytometry assay. As shown in Fig. 2C, nABP296 exhibited higher binding efficiency to MNNG/HOS than Cal27 cells, while nABP297 did not exhibit different binding efficiencies between MNNG/HOS or Cal27 cells. According to the results of the immunofluorescence assay, nABP296 co-localised with the CD105 antibody in the MNNG/HOS cell line and had no binding affinity for Cal27 cells (Fig. 2D). Furthermore, the ELISA experiment of nABP296 binding to CD105 and control proteins revealed that nABP296 had a higher affinity for CD105 protein in comparison with that for the control protein (Fig. 2E). Finally, nABP296 exhibited no cytotoxicity on the MNNG/HOS cell line after 24 and 48 h (Fig. 2F). Taken together, nABP296 can selectively bind to the CD105-positive MNNG/HOS cell line and is biocompatible with this cell line, suggesting that nABP296 may be able to target human osteosarcoma in vivo. The chemical structures of nABP296 and the HPLC/mass spectrometry results are shown in Fig. 3A and B, respectively.
Table II. Binding affinity of M13 phage for CD105 protein.

\begin{tabular}{lcc}
\hline $\begin{array}{l}\text { M13 } \\
\text { monoclonal phages }\end{array}$ & $\begin{array}{c}\text { Human recombinant } \\
\text { CD105 protein }\end{array}$ & NC \\
\hline A & 0.481 & 0.371 \\
B & 0.642 & 0.574 \\
C & 0.651 & 0.593 \\
D & 0.603 & 0.507 \\
E & 0.572 & 0.611 \\
F & 0.541 & 0.512 \\
G & 0.388 & 0.326 \\
H & 0.355 & 0.330 \\
I & 0.632 & 0.521 \\
J & 0.395 & 0.293 \\
K & 0.429 & 0.336 \\
L & 0.517 & 0.461 \\
M & 0.427 & 0.376 \\
N & 0.521 & 0.456 \\
O & 0.569 & 0.460 \\
P & 0.436 & 0.335 \\
\hline
\end{tabular}

ELISA for the binding activity of 16 monoclonal M13 phages to human recombinant CD105 protein. A total of 13 monoclonal M13 phages were observed to have higher affinity for CD105 protein than the NC blocking buffer. Monoclonal phages with a difference in absorbance of $>0.05$ were selected for sequencing. NC, negative control.

In vitro labelling of osteosarcoma. Following incubation of osteosarcoma histological sections derived from an MNNG/HOS xenograft tumour model and an osteosarcoma patient with nABP296, green fluorescence indicated nABP296 peptide and red flourescence indicated CD105 antibody. Results showed that the MNNG/HOS xenograft tumour model and osteosarcoma derived from the patient were successfully co-labelled with the nABP296 peptide and CD105 antibody (Fig. 4A and B). By contrast, absence of CD105 antibody and only weak nABP296 fluorescence were observed in the tongue carcinoma section (Fig. 4C). The results indicated that nABP296 was able to bind to osteosarcoma derived from a xenograft tumour model and a patient in vitro. Thus, nABP296 may be used in the diagnosis of osteosarcoma in place of CD105 in certain situations.

In vivo imaging of MNNG/HOS tumour. As shown in Fig. $5 \mathrm{~A}$, at $1 \mathrm{~h}$ after intravascular administration of nABP296 to MNNG/HOS tumour-bearing mice, fluorescence was detected in the MNNG/HOS xenograft tumour. Fluorescence detected in the tumour was higher compared with that in the surrounding tissues, whereas there was no fluorescence detected in the NC group. Furthermore, ex vivo imaging of tumours that were excised from the MNNG/HOS tumour-bearing mice was performed at $1.5 \mathrm{~h}$. It was observed that FITC-labelled nABP296 was accumulated in the 
A

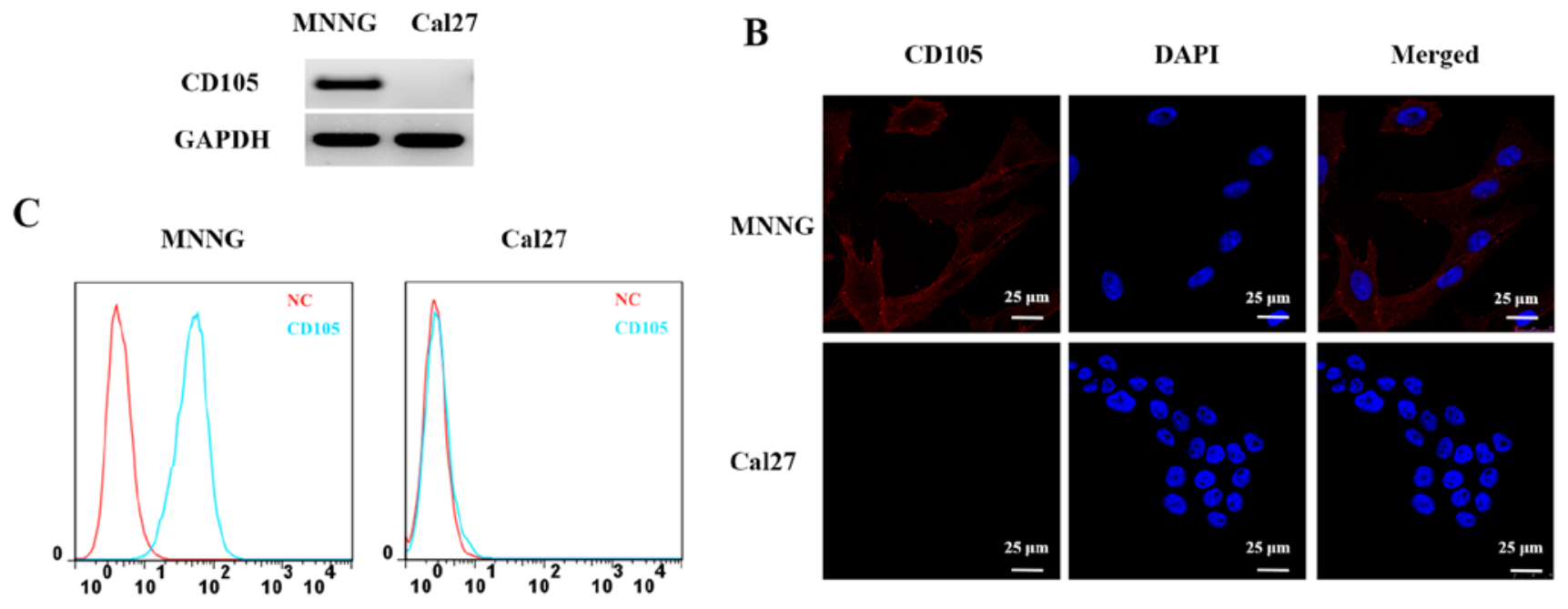

Figure 1. (A) Semiquantitative reverse transcription-polymerase chain reaction analysis of CD105 levels in MNNG/HOS and Ca127 cell lines. CD105 mRNA expression was observed in MNNG/HOS cells, whereas there was no CD105 mRNA expression in Cal27 cells. (B) Immunofluorescence analysis of CD105 expression in MNNG/HOS and Cal27 cell lines. As shown by red fluorescence, CD105 was located on the surface of MNNG/HOS cells. There was no CD105 fluorescence detected in the Cal27 cell line. (C) Flow cytometry analysis of CD105 expression in MNNG/HOS and Cal27 cell lines, confirming that MNNG/HOS cells highly expressed CD105 protein, while this was not expressed in Cal27 cells.

A

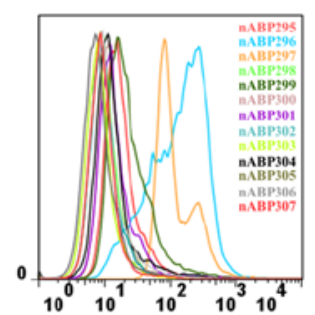

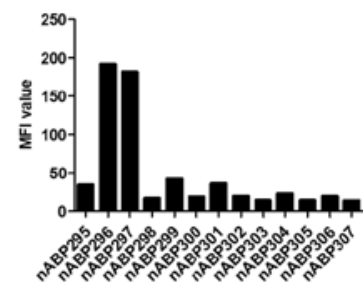

C

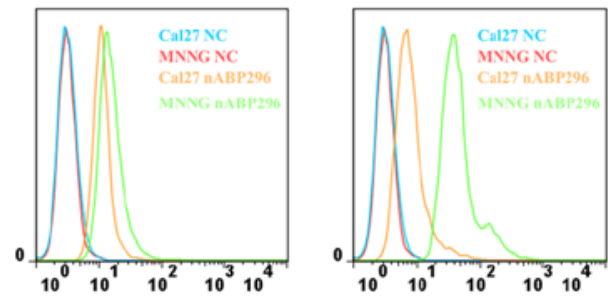

D

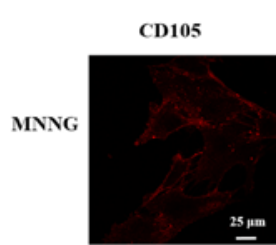

Cal27

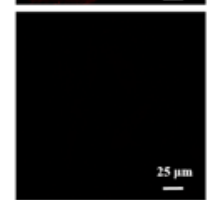

nABP296
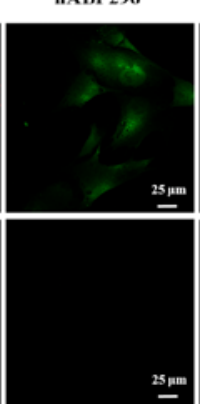

DAPI

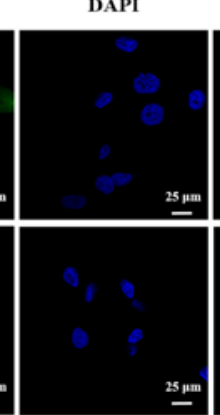

Merged
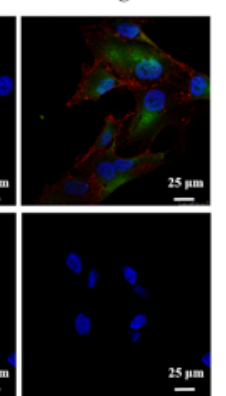

$\mathbf{E}$

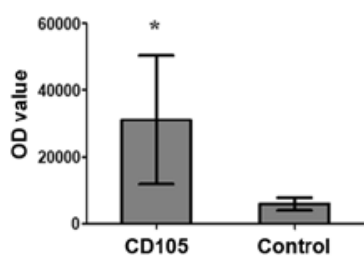

Figure 2. (A) Flow cytometry analysis of the binding affinity of 13 peptides for MNNG/HOS cells. nABP296 and nABP297 peptides exhibited higher binding affinity for the MNNG/HOS cell line. (B) MFI analysis of the flow cytometry results, demonstrating that nABP296 and nABP297 had the highest MFI. (C) Flow cytometry analysis of nABP296 and nABP297 in MNNG/HOS and Cal27 cells. nABP297 exhibited no binding affinity difference between MNNG/HOS and Ca127 cell lines, while nABP296 had a higher binding affinity for MNNG/HOS cells and lower affinity for Cal27 cells. Thus, nABP296 could be used for MNNG/HOS labelling in vitro and in vivo. (D) Immunofluorescence analysis of nABP296 in MNNG/HOS and Cal27 cells. nABP296 was co-located with the CD105 antibody in MNNG/HOS cells, whereas both CD105 and nABP296 exhibited no binding to Cal27. (E) ELISA of nABP296 binding affinity for CD105 protein, with CD106 protein used as control. The OD value in the CD105 protein group was higher than that in the CD106 protein group ( $\mathrm{P}<0.05$ vs. control). (F) Cytotoxicity assay of nABP296 on MNNG/HOS cells at 24 and 48 h, exhibiting no cytotoxicity on the MNNG/HOS cell line $(\mathrm{P}>0.05)$. nABP, non-antibody-binding protein; MFI, mean fluorescence intensity; OD, optical density.

xenograft tumour (Fig. 5B). Fluorescence was detected in frozen tumour histological sections, while there was no FITC fluorescence in the heart sections (Fig. 5C). These results supported the high affinity and specificity for nABP296 peptide for the identified MNNG/HOS xenograft tumour in vivo. 
Table III. Sequences of 13 clones obtained from screening that exhibited a positive ELISA result, and their characteristics.

\begin{tabular}{|c|c|c|c|c|}
\hline No. & Sequence & Molecular weight & Net charge & Protein-binding potential, $\mathrm{kcal} / \mathrm{mol}$ \\
\hline nABP295 & NWTTLSRSVNWP & $1,460.6$ & +1 & 2.21 \\
\hline nABP296 & WIYDTTRVIVPG & $1,419.6$ & 0 & 0.64 \\
\hline nABP297 & HAMSPVFLSKYA & $1,350.6$ & +1 & -0.06 \\
\hline nABP298 & LVPSILGATFIH & $1,267.5$ & 0 & -1.56 \\
\hline nABP299 & DISASLQSNRWH & $1,413.5$ & 0 & 3.05 \\
\hline nABP300 & AAGTFLMSMMSR & $1,302.6$ & +1 & 0.39 \\
\hline nABP301 & NNLPTSRTLAGN & $1,257.4$ & +1 & 2.56 \\
\hline nABP302 & GNNPLHVHHDKR & $1,423.6$ & +1 & 3.87 \\
\hline nABP303 & HHLRIPYALDQT & $1,463.7$ & 0 & 2.05 \\
\hline nABP304 & GTGAALAKVSEA & $1,074.2$ & 0 & 0.02 \\
\hline nABP305 & IKPVRALYTLAD & $1,359.6$ & +1 & 0.78 \\
\hline nABP306 & GTIRTSFWHTNT & $1,420.5$ & +1 & 2.39 \\
\hline nABP307 & GVHSVFAPLTPN & $1,238.4$ & 0 & -0.12 \\
\hline
\end{tabular}

nABP, non-antibody-binding protein.
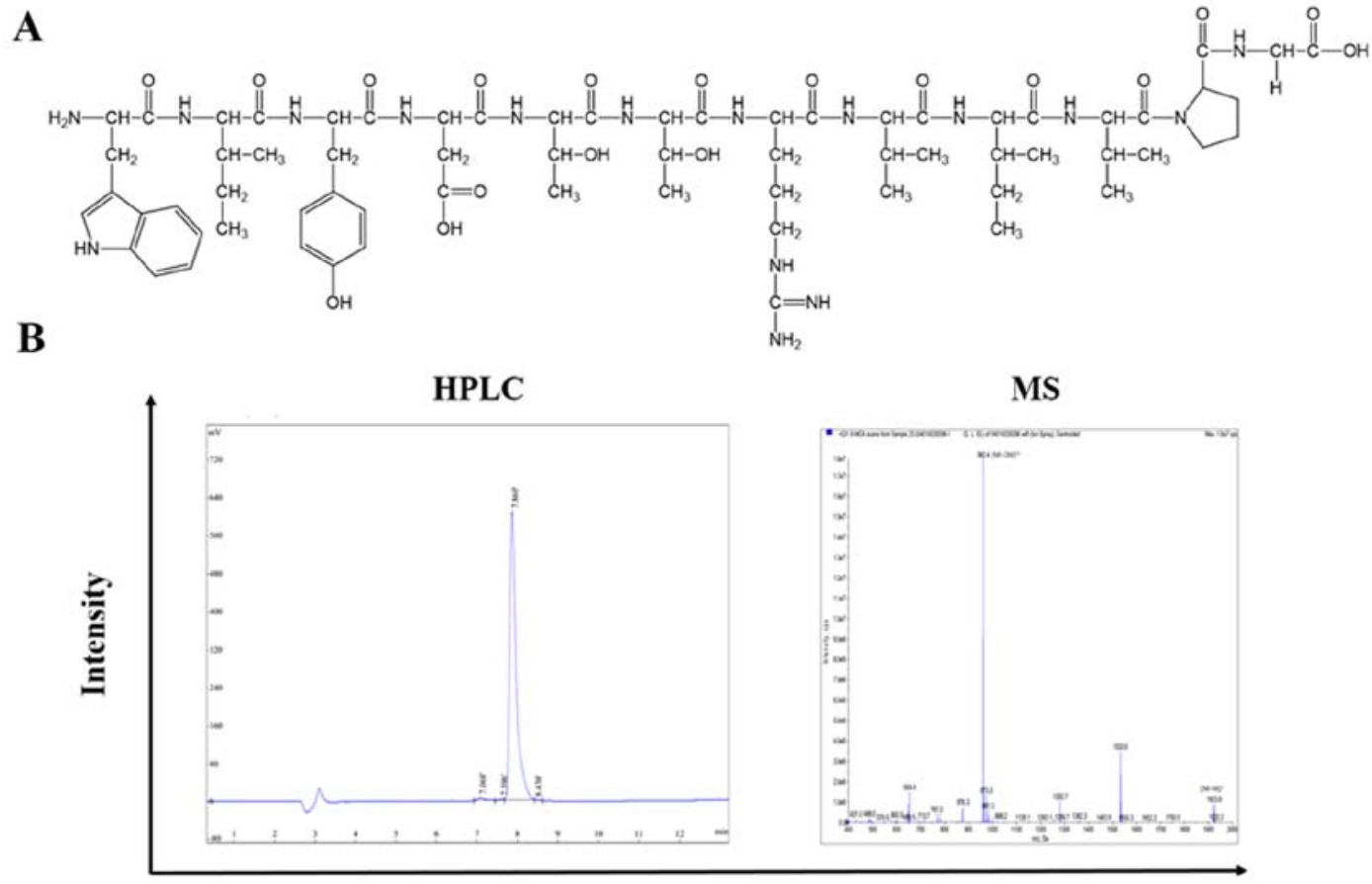

Time

Figure 3. (A) Chemical structure of nABP296. (B) High-performance liquid chromatography and mass spectrometry analysis of nABP296 peptide. nABP, non-antibody-binding protein.

\section{Discussion}

In the present study, it was demonstrated that nABP296, a 12 amino acid peptide, can specifically bind to recombinant human CD105 protein (Table II, and Fig. 2C-E). CD105 is a biomarker of MSCs that is used for the isolation and enrichment of these cells. The CD105 antibody has previously been used to isolate $\mathrm{CD} 105^{+}$phenotype MSCs from human cardiac mesenchymal stromal cells to enhance the function of the post-infarction heart in mice (20). In addition, the CD105 antibody is a reliable marker to distinguish the polydirectional differentiation potential of MSCs $(21,22)$. The nABP296 peptide discovered in the present study is relatively short and can specifically bind to the CD105-positive MNNG/HOS cell line. nABP296 was not found to have a cytotoxic effect on MNNG/HOS and Cal27 cells (Fig. 2E). Thus, nABP296 has the characteristics of specific affinity and good biocompatibility that can solve the problems of the usage of the CD105 antibody in vivo, and has the potential to serve as a novel probe to isolate MSCs from tissues. 
$\mathbf{A}$

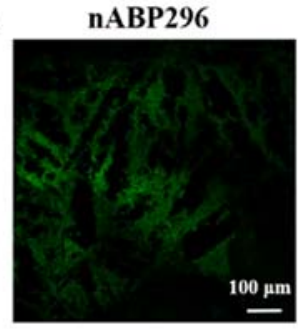

B

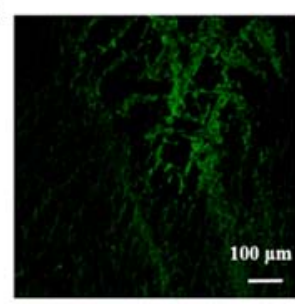

C

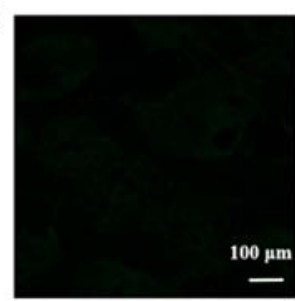

CD105
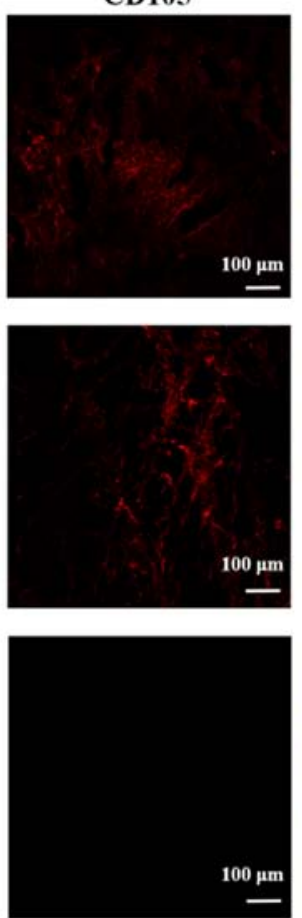

DAPI
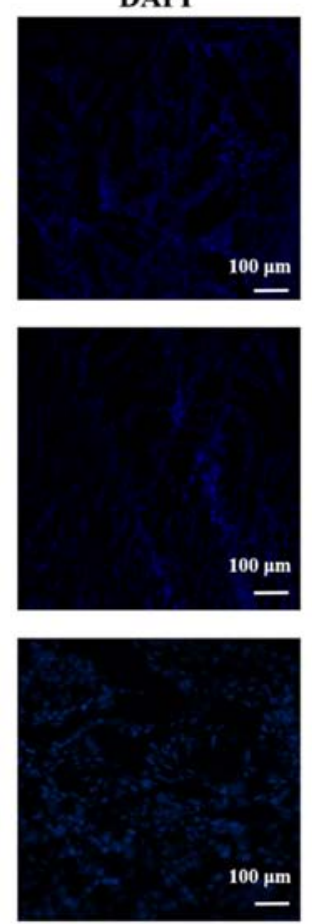

Merged
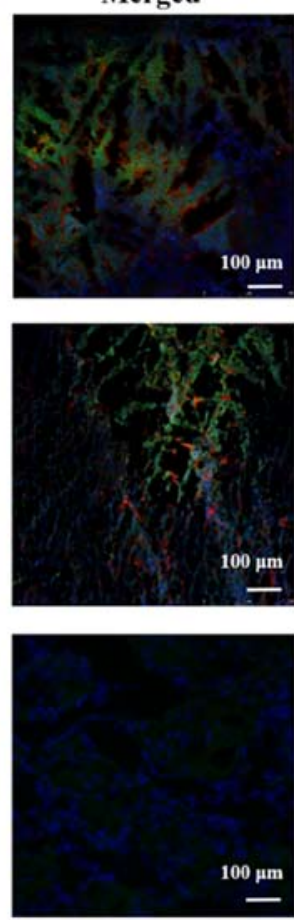

Figure 4. In vitro visualisation assay. (A) nABP296 exhibited colocalization with CD105 antibody in tumour sections derived from MNNG/HOS tumour-bearing mice. (B) A tumour histological section derived from an osteosarcoma patient could be label by nABP296 peptide and CD105 antibody. (C) Tumour histological section derived from an oral carcinoma patient, in which there was no CD105 antibody fluorescence and low nABP296 fluorescence. nABP, non-antibody-binding protein.

A

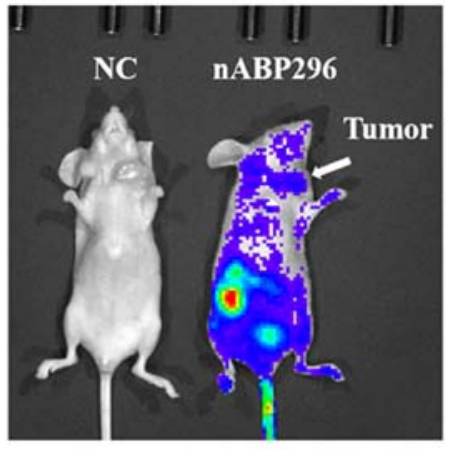

C
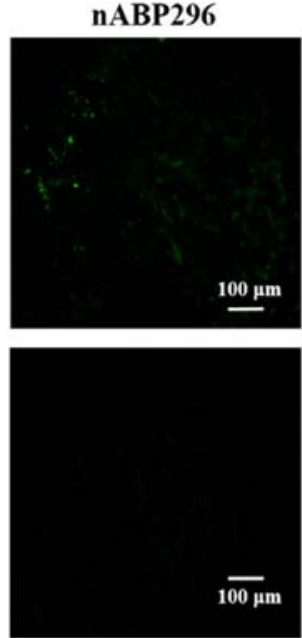

B

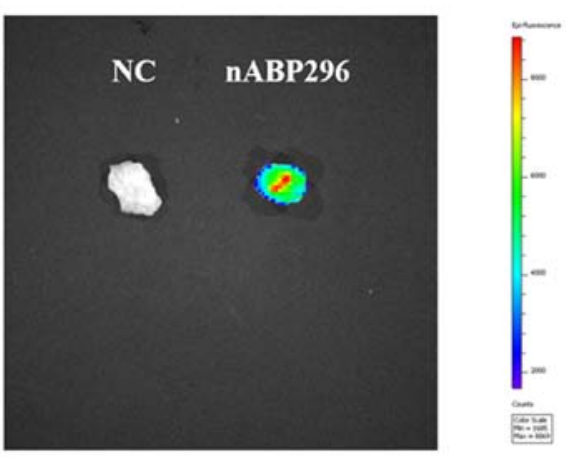

DAPI
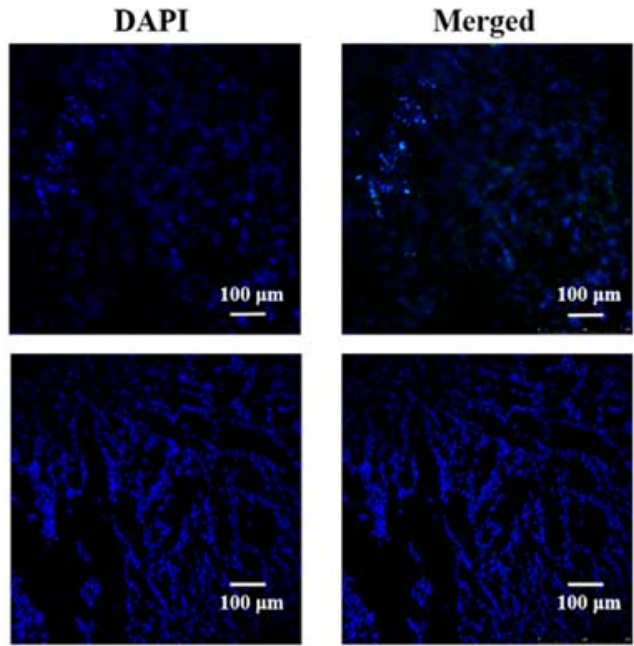

Figure 5. In vivo and ex vivo imaging of MNNG/HOS tumour-bearing mouse. (A) In vivo imaging of a MNNG/HOS tumour-bearing mouse at $1 \mathrm{~h}$ after intravenous administration of nABP296 peptide. Fluorescence was detected in the area of the liver, kidneys and xerograph tumour. (B) Ex vivo imaging of tumours that were excised from the MNNG/HOS tumour-bearing mouse at $1.5 \mathrm{~h}$, demonstrating that FITC-labelled nABP296 was accumulated in the xerograph tumour. (C) Frozen sections of organs excised from MNNG/HOS tumour-bearing mice. FITC-labelled nABP296 was detected in the tumour histological sections, while there was no fluorescence in the heart section. The results demonstrated that nABP296 was accumulated in the MNNG/HOS xerograph tumour. nABP, non-antibody-binding protein. 
Angiogenesis, the process of new blood vessel formation in tissues, is necessary for tumour growth and metastasis. CD105 serves a crucial role in angiogenesis (23) and is thus overexpressed in actively proliferating tumours $(24,25)$. In vivo targeting by CD105 can be used for tumour positron-emission tomography imaging and visualization. In a previous study, CD105 labelled with ${ }^{64} \mathrm{Cu}$ was used in BXPC-3 xenograft tumour imaging (26). CD105 conjugated with IRDye $800 \mathrm{CW}$ was used to label 4T1 tumour-bearing mice, which were then visualised by near-infrared fluorescence imaging (27). Peptides have been used for osteosarcoma imaging in numerous studies. Ma et al (28) reported that several peptides derived from a T7 phage display were used for in vivo specific photoacoustic imaging of osteosarcomas. The contrast of osteosarcoma images was enhanced by $170-230 \%$. Other peptides, including the hydroxyapatite binding peptide, were used to characterise hydroxyapatite on human osteotropic tumour tissue (29). OSP-1 derived from the Ph.D.-12 Phage Display Peptide Library was able to specifically bind to $143 \mathrm{~B}$ osteosarcomas, other than 293T human embryonic kidney cells. The binding site of OSP-1 may be associated with heparin sulphate proteoglycans (30). In the present study, it was reported that nABP296 was able to locate osteosarcomas in MNNG/HOS tumour-bearing mice (Fig. 5). The nABP296 peptide is a relatively short, human-made peptide that is synthesised in vitro, and has no animal heterologous form or immune response. nABP296 is nontoxic to cells, thus, it can be safely used in humans. This peptide can be activated by fluorescence, and it is therefore useful for the detection of tumour cells. Owning to these advantages, the nABP296 peptide may be a new strategy for the early diagnosis and detection of metastasis of osteosarcoma in patients.

nABPs usually consist of $<50$ amino acids, and their penetrating ability to the tumour microenvironment is superior to that of antibodies. Owing to the potent penetrating ability and targeting effect, peptides have been used as cargo in antitumour therapy. Peptides favoured the cellular absorption of Ara-C in Caco-2 cells to increase their internalization rate (31). In a study by Ma et al (32), an $\alpha \mathrm{v} \beta 3$-targeting peptide conjugated with methotrexate was demonstrated to increase the efficacy of methotrexate and reduce its side effects. In the immunofluorescence assay conducted in the present study, nABP296 was detected in the cytoplasm, suggesting that nABP296 was able to penetrate the cell membrane. Thus, nABP296 may serve as a CD105 protein target cargo to transfer antitumour drugs to CD105-positive tumour cells or therapeutic agents to target cells.

Future research must focus on the targeting properties of nABP296. In addition, the mechanism underlying the penetrating effect of nABP296 remains unclear and needs to be determined. Based on the aforementioned findings, nABP296 may serve as a targeted therapeutic cargo conjugate with other therapeutic agents.

In conclusion, in the present study, a novel peptide, namely nABP296, was discovered by M13 phage biopanning, and this peptide specifically binds to the recombinant human CD105 protein. nABP296 labels frozen osteosarcoma sections derived from patients and an MNNG/HOS xenograft tumour model in vitro. In addition, the MNNG/HOS xenograft tumour model was labelled by nABP296 in vivo. Therefore, this peptide may be used for diagnosis and osteosarcoma imaging in vivo, as well as for MSC isolation and CD105 targeted therapy in the future.

\section{Acknowledgements}

Not applicable.

\section{Funding}

The authors acknowledge the financial supportfrom the National Natural Science Foundation of China (grant no. 31371390), the Doctoral Foundation of Ministry of Education of China (grant no. 20130171110010), the Program of the State High-Tech Development Project (grant no. 2014AA020702) and the Program of Guangdong Science and Technology (grant nos. 2016B030231001 and 2017B020230002).

\section{Availability of data and materials}

The datasets used and/or analysed in the present study are available from the corresponding author on reasonable request.

\section{Authors' contributions}

XL and HW conceived and designed the experiments. XL and $\mathrm{XH}$ performed the experiments. JZ, HH and LZ analysed the data. XL and HW wrote the manuscript. MY and YZ reviewed and polished the paper.

\section{Ethics approval and consent to participate}

The animal use protocol was reviewed and approved by the Animal Ethical and Welfare Committee of Sun Yat-sen University. The described patient experiments were approved by the Ethics Committee of The Hospital of Stomatology at Sun Yat-sen University (approval no. ERC-2017-11). All patients participating in this study provided writtem informed consent prior to tissue collection and agreed to the use of their samples in scientific research.

\section{Patient consent for publication}

All patients participating in this study provided written informed consent.

\section{Competing interests}

The authors declare no conflict of interest. The founding sponsors had no role in the design of the study; in the collection, analyses, or interpretation of data; in the writing of the manuscript, and in the decision to publish the results.

\section{References}

1. Li C, Hampson IN, Hampson L, Kumar P, Bernabeu C and Kumar S: CD105 antagonizes the inhibitory signaling of transforming growth factor beta1 on human vascular endothelial cells. FASEB J 14: 55-64, 2000.

2. Gougos A and Letarte M: Primary structure of endoglin, an RGD-containing glycoprotein of human endothelial cells. J Biol Chem 265: 8361-8364, 1990. 
3. Barbara NP, Wrana JL and Letarte M: Endoglin is an accessory protein that interacts with the signaling receptor complex of multiple members of the transforming growth factor-beta superfamily. J Biol Chem 274: 584-594, 1999.

4. Li C, Guo B, Wilson PB, Stewart A, Byrne G, Bundred N and Kumar S: Plasma levels of soluble CD105 correlate with metastasis in patients with breast cancer. Int J Cancer 89: 122-126, 2000.

5. Duff SE, Li C, Garland JM and Kumar S: CD105 is important for angiogenesis: Evidence and potential applications. FASEB J 17: 984-992, 2003.

6. Marioni G, D'Alessandro E, Giacomelli L and Staffieri A: CD105 is a marker of tumour vasculature and a potential target for the treatment of head and neck squamous cell carcinoma. J Oral Pathol Med 39: 361-367, 2010.

7. Minhajat R, Mori D, Yamasaki F, Sugita Y, Satoh T and Tokunaga O: Organ-specific endoglin (CD105) expression in the angiogenesis of human cancers. Pathol Int 56: 717-723, 2006.

8. Muniz C, Teodosio C, Mayado A, Amaral AT, Matarraz S, Barcena P, Sanchez ML, Alvarez-Twose I, Diez-Campelo M, Garcia-Montero AC, et al: Ex vivo identification and characterization of a population of $\mathrm{CD} 13^{\text {high }} \mathrm{CD} 105^{+} \mathrm{CD} 45^{-}$mesenchyma stem cells in human bone marrow. Stem Cell Res Ther 6: 169 2015.

9. Odabas S, Sayar F, Guven G, Yanikkaya-Demirel G and Piskin E: Separation of mesenchymal stem cells with magnetic nanosorbents carrying CD105 and CD73 antibodies in flow-through and batch systems. J Chromatogr B Analyt Technol Biomed Life Sci 861: 74-80, 2008.

10. Cavar S, Jelasic D, Seiwerth S, Milosevic M, Hutinec Z and Misic M: Endoglin (CD 105) as a potential prognostic factor in neuroblastoma. Pediatr Blood Cancer 62: 770-775, 2015.

11. Zhou Y, Gu H, Xu Y, Li F, Kuang S, Wang Z, Zhou X, Ma H, Li P, Zheng Y, et al: Targeted antiangiogenesis gene therapy using targeted cationic microbubbles conjugated with CD105 antibody compared with untargeted cationic and neutral microbubbles. Theranostics 5: 399-417, 2015.

12. Damron TA, Ward WG and Stewart A: Osteosarcoma, chondrosarcoma, and Ewing's sarcoma: National cancer data base report. Clin Orthop Relat Res 459: 40-47, 2007.

13. Savage SA and Mirabello L: Using epidemiology and genomics to understand osteosarcoma etiology. Sarcoma 2011: 548151, 2011.

14. Allison DC, Carney SC, Ahlmann ER, Hendifar A, Chawla S, Fedenko A, Angeles C and Menendez LR: A meta-analysis of osteosarcoma outcomes in the modern medical era. Sarcoma 2012: 704872, 2012.

15. Gibbs CP, Kukekov VG, Reith JD, Tchigrinova O, Suslov ON Scott EW, Ghivizzani SC, Ignatova TN and Steindler DA: Stem-like cells in bone sarcomas: Implications for tumorigenesis. Neoplasia 7: 967-976, 2005.

16. Reubi JC: Peptide receptors as molecular targets for cancer diagnosis and therapy. Endocr Rev 24: 389-427, 2003.

17. Xiang Z, Yang X, Xu J, Lai W, Wang Z, Hu Z, Tian J, Geng L and Fang Q: Tumor detection using magnetosome nanoparticles functionalized with a newly screened EGFR/HER2 targeting peptide. Biomaterials 115: 53-64, 2017.
18. Alexander-Bryant AA, Zhang H, Attaway CC, Pugh W, Eggart L, Sansevere RM, Andino LM, Dinh L, Cantini LP and Jakymiw A: Dual peptide-mediated targeted delivery of bioactive siRNAs to oral cancer cells in vivo. Oral Oncol 72: 123-131, 2017.

19. Ma Y, Zhao S, Shen S, Fang S, Ye Z, Shi Z and Hong A: A novel recombinant slow-release TNF $\alpha$-derived peptide effectively inhibits tumor growth and angiogensis. Sci Rep 5: 13595, 2015.

20. Czapla J, Matuszczak S, Wisniewska E, Jarosz-Biej M, Smolarczyk R, Cichon T, Glowala-Kosinska M, Sliwka J, Garbacz M, Szczypior M, et al: Human cardiac mesenchymal stromal cells with $\mathrm{CD} 105^{+} \mathrm{CD} 34$ phenotype enhance the function of post-infarction heart in mice. PLoS One 11: e158745, 2016

21. Yamamoto M, Nakata H, Hao J, Chou J, Kasugai S and Kuroda S: Osteogenic potential of mouse adipose-derived stem cells sorted for CD90 and CD105 in vitro. Stem Cells Int 2014: 576358, 2014

22. Fan W, Li J, Wang Y, Pan J, Li S, Zhu L, Guo C and Yan Z CD105 promotes chondrogenesis of synovium-derived mesenchymal stem cells through Smad2 signaling. Biochem Biophys Res Commun 474: 338-344, 2016.

23. Li DY, Sorensen LK, Brooke BS, Urness LD, Davis EC, Taylor DG, Boak BB and Wendel DP: Defective angiogenesis in mice lacking endoglin. Science 284: 1534-1537, 1999.

24. Takase Y, Kai K, Masuda M, Akashi M and Tokunaga O Endoglin (CD105) expression and angiogenesis status in small cell lung cancer. Pathol Res Pract 206: 725-730, 2010.

25. Miyata Y, Mitsunari K, Asai A, Takehara K, Mochizuki Y and Sakai H: Pathological significance and prognostic role of microvessel density, evaluated using CD31, CD34, and CD105 in prostate cancer patients after radical prostatectomy with neoadjuvant therapy. Prostate 75: 84-91, 2015.

26. Luo H, England CG, Shi S, Graves SA, Hernandez R, Liu B, Theuer CP, Wong HC, Nickles RJ and Cai W: Dual targeting of tissue factor and CD105 for preclinical PET imaging of pancreatic cancer. Clin Cancer Res 22: 3821-3830, 2016.

27. Zhang Y, Hong H, Severin GW, Engle JW, Yang Y, Goel S, Nathanson AJ, Liu G, Nickles RJ, Leigh BR, et al: ImmunoPET and near-infrared fluorescence imaging of CD105 expression using a monoclonal antibody dual-labeled with ${ }^{89} \mathrm{Zr}$ and IRDye 800CW. Am J Transl Res 4: 333-346, 2012.

28. Ma Z, Qin H, Chen H, Yang H, Xu J, Yang S, Hu J and Xing D: Phage display-derived oligopeptide-functionalized probes for in vivo specific photoacoustic imaging of osteosarcoma. Nanomedicine 13: 111-121, 2017.

29. Lee JS and Tung CH: Osteotropic cancer diagnosis by an osteocalcin inspired molecular imaging mimetic. Biochim Biophys Acta 1830: 4621-4627, 2013.

30. Sun X, Niu G, Yan Y, Yang M, Chen K, Ma Y, Chan N, Shen B and Chen X: Phage display-derived peptides for osteosarcoma imaging. Clin Cancer Res 16: 4268-4277, 2010.

31. Cheon EP, Hong JH and Han HK: Enhanced cellular uptake of Ara-C via a peptidomimetic prodrug, L-valyl-ara-C in Caco-2 cells. J Pharm Pharmacol 58: 927-932, 2006.

32. Ma P, Yu H, Zhang X, Mu H, Chu Y, Ni L, Xing P, Wang Y and Sun K: Increased active tumor targeting by an $\alpha v \beta 3$-targeting and cell-penetrating bifunctional peptide-mediated dendrimer-based conjugate. Pharm Res 34: 121-135, 2017. 